\title{
Expressions and clinic significance of miRNA-143, miRNA- 34A, miRNA-944, miRNA-101 and miRNA-218 in cervical cancer tissues
}

\author{
Feng Wang ${ }^{1,2}$, Qing-Wei Zhang ${ }^{2}$, Xiu-Hong $\mathrm{Fu}^{2}$, Hui-Fen Wang ${ }^{2}$ and $\mathrm{Yu}$-Ling \\ Liu $^{1 *}$ \\ ${ }^{1}$ Department of Obstetrics and Gynaecology, The Second Affiliated Hospital of Zhengzhou University, Zhengzhou 450014, \\ ${ }^{2}$ Department of Obstetrics and Gynaecology, The First Affiliated Hospital of Luohe Medical College, Luohe 462000, China \\ *For correspondence: Email: liuyulingzz@163.com; Tel/Fax: +86-0395-3356036
}

\begin{abstract}
Purpose: To search for novel biomarkers for early diagnosis of cervical cancer, as well as novel therapeutic target for cervical cancer.

Methods: A total of 96 cervical tissue specimens were collected from patients in the Second Affiliated Hospital of Zhengzhou University, out of which 10 were normal control. The remaining specimens (86) were cervical cancer specimens and were divided into 4 groups ( $A$ - D) based on tumor-biomarker levels of CA125 and SCC. Quantitative real-time polymerase chain reaction technology (qRT-PCR) was used to detect the expressions of miRNA-143, miRNA-34A, miRNA-944, miRNA-101 and miRNA-218 in the cervical cancer tissues.

Results: The levels of CA125 (U/mL) and SCC (ug/L) expressed in normal control group and groups A $D$ were 11.75 and $0.73(n=10), 382$ and $2.72(n=25), 912.9$ and $3.93(n=21), 1675$ and $5.87(n=29)$, and 2120 and $6.66(n=11)$, respectively. Furthermore, qRT-PCR results showed that the expressions of miRNA-944 and miRNA-218 in cervical cancer tissues were markedly up-regulated compared to normal control tissues $(p<0.01)$. In contrast, the expression level of miRNA-143, miRNA-34A, and miRNA-101 were significantly decreased $(p<0.01)$.

Conclusion: The biomarkers, miRNA-143, miRNA-34A, miRNA-944, miRNA-101 and miRNA-218, can be considered novel for early diagnosis of cervical cancer.
\end{abstract}

Keywords: Cervical cancer, Biomarkers, miRNA-143, miRNA-34A, miRNA-944, miRNA-101, miRNA218

Tropical Journal of Pharmaceutical Research is indexed by Science Citation Index (SciSearch), Scopus, International Pharmaceutical Abstract, Chemical Abstracts, Embase, Index Copernicus, EBSCO, African Index Medicus, JournalSeek, Journal Citation Reports/Science Edition, Directory of Open Access Journals (DOAJ), African Journal Online, Bioline International, Open-J-Gate and Pharmacy Abstracts

\section{INTRODUCTION}

Cervical cancer is a serious threat to the health of women, and is one of the leading causes of cancer-related death in the world due to delayed diagnosis and high risk of metastasis [1]. Furthermore, $85 \%$ of the cervical cancers occur in developing countries, such as China and India [2]. Currently, chemotherapy is the commonly used strategy to prevent the relapse and metastasis of cervical cancer besides surgery [3]. However, early diagnosis is the most important strategy for treating cervical cancer [4].

MicroRNAs (miRNAs) are a class of non-coding RNAs (21 - 24 nucleotides in length) that are critical for many important processes such as development, differentiation and even 
carcinogenesis, and can regulate the chemo sensitivity of tumor cells [5,6]. Accumulating evidence has demonstrated that dysregulation of miRNAs which function as tumor promoter or suppressor depending on the nature of its targets, occurs in various human cancers [7].

Currently, miRNAs involved in carcinogenesis and progression of cervical cancer have been widely investigated [8]. In our present study, we investigated the expressions of miRNA-143, miRNA-34A, miRNA-944, miRNA-101 and miRNA-218 in cervical cancer tissues, and explored their regulative significance on cervical cancer tissues. Our research on miRNAs is aimed to provide reference for the early clinical diagnosis of cervical cancer and provide novel therapeutic target for cervical cancer.

\section{EXPERIMENTAL}

\section{Subjects and sample collection}

A total of 86 fresh cervical tissue specimens were obtained from patients treated in the Second Affiliated Hospital of Zhengzhou University for squamous cell carcinoma. Additionally, normal cervical tissue specimens (group A) were also obtained from patients without cervical cancer $(n=10)$. The 86 cervical cancer patients were divided into 4 groups ( $B$ E) based on the tumor-biomarker levels of CA125 and SCC, and the numbers of subjects were 25, 21, 29 and 10, respectively. All the patients enrolled were required to read and sign an informed consent form voluntarily before enrollment. All the experimental designs were carried out in accordance with the declaration of Helsinki promulgated in 1964 as amended in 1996 [9] and approved by the Ethics Committee of the Second Affiliated Hospital of Zhengzhou University (Ethical approval No.: S2013-06-23).

\section{RNA extraction and reverse transcription}

Total RNA was extracted from each of the experimental groups using Trizol Reagent (Shanghai Sangon, Shanghai, China) according to the manufacturer's instructions. Reverse transcription (RT) was performed on RNA samples, followed by polymerase chain reaction (PCR) amplification.

\section{Quantitative real-time polymerase chain reaction (qRT-PCR)}

Quantitative RT-PCR was performed to detect the relative transcript levels of miRNA-143, miRNA-34A, miRNA-944, miRNA-101 and miRNA-218, respectively. PCR was performed under the following conditions: $94{ }^{\circ} \mathrm{C}$ for $4 \mathrm{~min}$ followed by 40 cycles of $94{ }^{\circ} \mathrm{C}$ for $1 \mathrm{~min}, 56{ }^{\circ} \mathrm{C}$ for $1 \mathrm{~min}$ and $72{ }^{\circ} \mathrm{C}$ for $1 \mathrm{~min}$. U6 snRNA was used as an endogenous control to normalize the expression of miRNA-143, miRNA-34A, miRNA944, miRNA-101 and miRNA-218, respectively. qRT-PCR was performed using FastStart Universal SYBR Green Master kit (Roche Diagnostics) and analyzed with an Applied Biosystems 7900 Real-Time PCR System. The specific primer pairs (Invitrogen, Shanghai, China) are shown in Table 1.

\section{Statistical analysis}

All the data are presented as mean \pm standard deviation (SD) and group comparison was performed by ANOVA (SPSS 16.0). $P<0.05$ was considered statistically significant.

\section{RESULTS}

\section{Clinicopathologic characteristics of subjects}

Ten normal women and eighty six women with cervical cancer were included in the study.

Table 1: Primers used for quantitative real-time PCR

\begin{tabular}{lll}
\hline & & \\
\hline U6 & F & 5'GCTTCGGCAGCACATATACTAAAAT3' \\
miRNA-143 & R & 5'CGCTTCACGATTTGCGTGTCAT3' \\
& F & GGGCGGGTGCAGTGCT \\
miRNA-34A & F & GTCGTATCCAGTGCGTGTCGTGGAGTCGGCAATTGCACTGGATACGACACCAGA \\
& R & GTCGTATCCAGTGCGTGTCGTGGAGTCGGCAATTGCACTGGATACGACACAACC \\
miRNA-944 & F & GGGCGGAAATTATTGTA \\
& R & GTCGTATCCAGTGCGTGTCGTGGAGTCGGCAATTGCACTGGATACGACCTCATCC \\
miRNA-101 & F & GGGCGGCAGTTATCACAG \\
& R & GTCGTATCCAGTGCGTGTCGTGGAGTCGGCAATTGCACTGGATACGACAGCATC \\
miRNA-218 & F & GGGCGGTTGTGCTTGAT \\
& R & GTCGTATCCAGTGCGTGTCGTGGAGTCGGCAATTGCACTGGATACGACATGGT \\
\hline
\end{tabular}


Basic characteristics of five groups are given in Table 2. A represented normal control, and B-E represented the 4 groups of cervical cancer patients divided according to the biomarker expression levels of cancer antigen 125 (CA125) and squamous cell carcinoma antigen (SCC). Overall, no obvious difference was observed for the height, age, weight, and fasting bloodglucose (FBG) in the five groups $(p>0.05)$. However, the values of CA125 and SCC in the 5 groups were significantly different. As shown in Table 2, the CA125 and SCC values of the 4 groups of patients with cervical cancer were significantly higher than the normal values of CA125.

\section{PCR results}

To further validate the altered expressions of miRNA-143, miRNA-34A, miRNA-944, miRNA-
101 and miRNA-218 in cervical cancer, we tested the expressions of miRNA-143, miRNA34A, miRNA-944, miRNA-101 and miRNA-218 in the cervical cancer tissues and normal tissues by using qRT-PCR assay. The amplification plots of qRT-PCR are shown in Figure 1. As shown in Table 3, miRNA-944 and miRNA-218 in the 4 groups of cervical cancer tissues were found to be expressed at significantly higher levels when compared to the normal control tissues $(p<$ 0.01). Meanwhile, the expression levels of miRNA-143, miRNA-34A, and miRNA-101 in the 4 groups of patients with cervical cancer were relatively down-regulated $(p<0.01)$. The results showed that the expressions of miRNA-944 and miRNA-218 changed dramatically with the serious extent of cervical cancer.

Table 2: Baseline demographics and disease characteristics

\begin{tabular}{lcccccc}
\hline Group & Height $(\mathbf{c m})$ & Age & Weight & FBG (mmol/L) & CA125 (U/mL) & SCC (ug/L) \\
\hline A & $159.91 \pm 4.65$ & $43.62 \pm 10.33$ & $53.41 \pm 4.17$ & $5.04 \pm 1.25$ & $11.75 \pm 2.98$ & $0.73 \pm 0.28$ \\
B & $162.76 \pm 4.54$ & $41.16 \pm 9.12$ & $55.04 \pm 6.19$ & $4.94 \pm 0.64$ & $382 \pm 97.29$ & $2.72 \pm 0.88$ \\
C & $162.76 \pm 3.97$ & $41.16 \pm 8.44$ & $55.04 \pm 6.13$ & $4.9412 \pm 0.93$ & $912.9 \pm 99.76$ & $3.93 \pm 0.93$ \\
D & $162.03 \pm 4.73$ & $42.03 \pm 8.26$ & $58.72 \pm 5.07$ & $4.69 \pm 0.89$ & $1675 \pm 246.78$ & $5.87 \pm 1.14$ \\
E & $160.82 \pm 5.40$ & $43.09 \pm 9.28$ & $53.91 \pm 4.83$ & $4.61 \pm 0.89$ & $2120 \pm 234.45$ & $6.66 \pm 1.28$ \\
\hline
\end{tabular}

Key: FBG: Fasting blood-glucose; A: normal control; B-E represented the 4 groups of cervical cancer patients, and cervical cancer patients were divided according to the biomarkers expression levels of CA125 and SCC
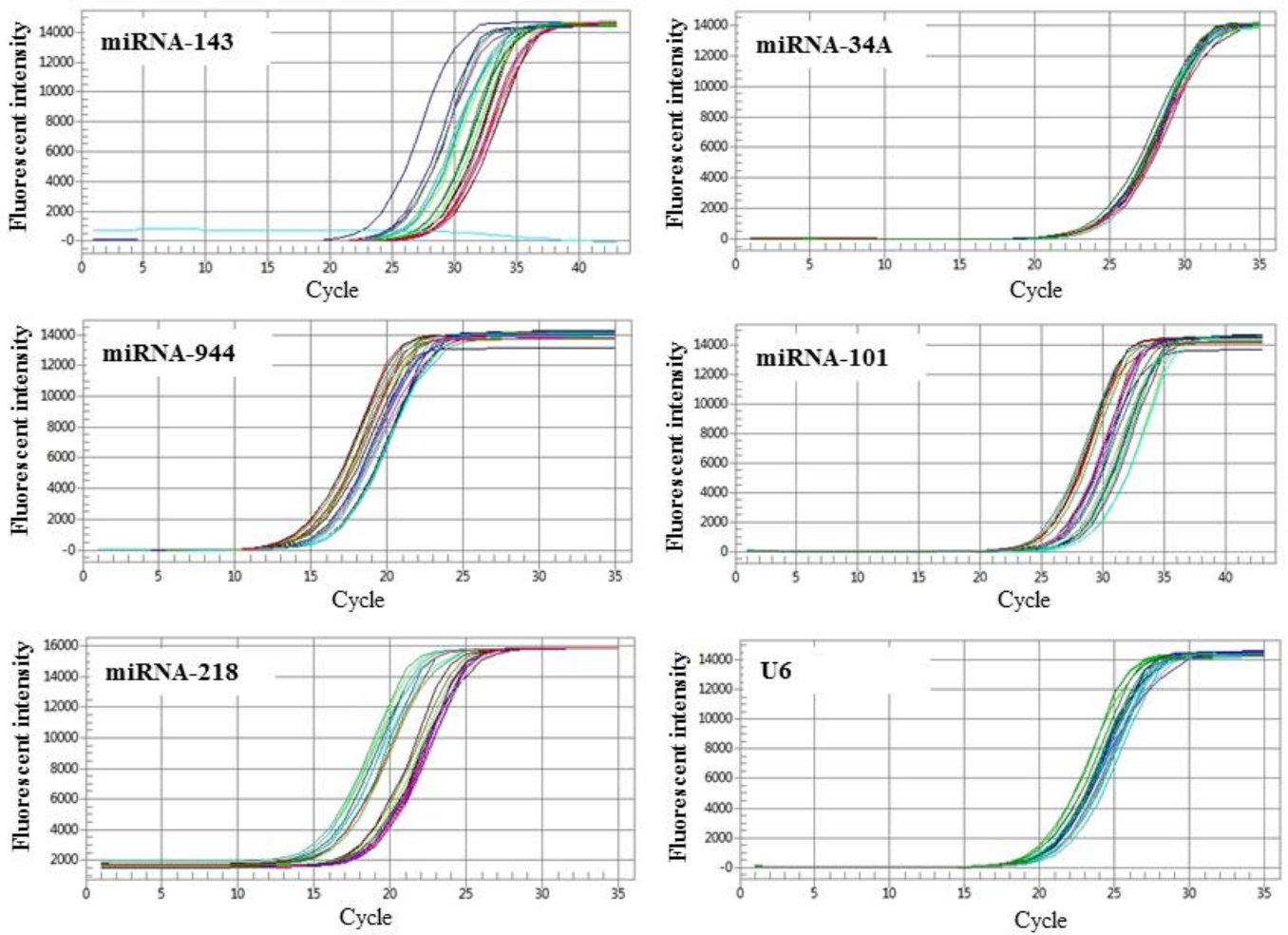

Figure 1: The amplification plots of real-time quantitative PCR. 
Table 3: Expressions of the miRNA-143, 34A, 944, 101 and 218 in patients with cervical cancer

\begin{tabular}{llll}
\hline & miRNA-143 & miRNA-34A & miRNA-944 \\
\hline A & $0.744863 \pm 0.156102$ & $0.725107 \pm 0.194532$ & $0.695712 \pm 0.190653$ \\
B & $0.000413 \pm 0.000113^{* *}$ & $0.092564 \pm 0.012031^{* *}$ & $25.22961 \pm 5.987026^{* *}$ \\
C & $0.000384 \pm 0.000124^{* *}$ & $0.087595 \pm 0.019765^{* *}$ & $27.50011 \pm 5.976513^{* *}$ \\
D & $0.000343 \pm 0.000123^{* *}$ & $0.065139 \pm 0.015563^{* *}$ & $32.41054 \pm 11.74653^{* *}$ \\
E & $0.000341 \pm 0.000115^{* *}$ & $0.063897 \pm 0.021022^{* *}$ & $39.55085 \pm 12.65385^{* *}$ \\
\hline \multicolumn{2}{l}{ miRNA-101 } & miRNA-218 \\
\hline A & $1.265348 \pm 0.344745$ & $0.732692 \pm 0.243097$ & \\
B & $0.373367 \pm 0.098217^{* *}$ & $21.18917 \pm 2.687207^{* *}$ & \\
C & $0.398324 \pm 0.176539^{* *}$ & $24.84102 \pm 5.210923^{* *}$ & \\
D & $0.448022 \pm 0.138603^{* *}$ & $25.87216 \pm 7.864375^{* *}$ & \\
E & $0.546068 \pm 0.176068 * *$ & $35.21287 \pm 7.412874 * *$ &
\end{tabular}

A: normal control; B-E represented the 4 groups of cervical cancer patients, and cervical cancer patients were divided according to the biomarkers expression levels of CA125 and SCC; ${ }^{* *}$ significant difference at $p<0.01$, respectively vs. normal control

\section{DISCUSSION}

In the present study, we systematically investigated the expression of miRNA-143, miRNA-34A, miRNA-944, miRNA-101 and miRNA-218 in cervical cancer tissues, and found that in cervical cancer tissues, miRNA-944 and miRNA-218 were dramatically up-regulated, whereas miRNA-143, miRNA-34A and miRNA101 were decreased.

A number of studies have identified miRNA-143 as a tumor suppressor gene lost expression significantly in several cancer types, including breast and cervical cancers [10-13], which is consistent with our results on the expression of miRNA-143 in cervical cancer tissues. miRNA143 was expressed at significantly lower levels when compared to the normal control tissues. In addition, miRNA-34A belongs to the miRNA-34 family and acts as a tumor suppressor in several cancer types, and ectopic miRNA-34 expression induces apoptosis, cell-cycle arrest or senescence [14,15]. Recently, many studies have reported that miRNA-34A was downregulated in many human cancers through hypermethylation of promoter DNA to silence this miRNA $[14,16]$. In the present study, miRNA-34A in patients with cervical cancer was also downregulated.

The gene coding for miRNA-944 is located in the intron of TP63 gene, which encodes tumor protein 63 (p63). And the expression of miRNA944 may correlate with TP63 expression [17]. miRNA-944, which targets an mRNA of SOCS (suppressor of cytokine signaling) family tumor suppressor genes, can promote tumor growth, proliferation, and squamous differentiation [18]. In addition, miRNA-944 was first identified in human cervical cells using a small RNA cloning approach and it was reported that it is significantly more abundant in cervical cancer tissues than their normal counterparts $[18,19]$. In the present study, the expression of miRNA-944 in cervical cancer tissues had significantly changed compared to other miRNAs and miRNA-944 was expressed at significantly higher levels compared to normal tissues. Thus, the miRNA-944 in cervical cancer tissues might be regarded as an important biomarker in the process of diagnosing cervical cancer.

miRNA-101 is one of the down-regulated miRNAs in a diverse of malignancies relative to their non-tumor counterparts, such as colorectal cancer, ovarian carcinoma, and non-small cell lung cancer, etc [20-22]. Furthermore, downregulated expression of miRNA-101 might be involved in the progression of malignant cancers [23]. The results show that the expression of miRNA-101 in patients with cervical cancer is down-regulated when compared with the normal tissues. As a broadly conserved miRNA, miR218 is considered a tumor suppressor in multiple carcinomas by targeting different genes, such as bladder cancer, liver cancer, and breast cancer, etc [24-26].

Many researchers have reported that the expression of miRNA-218 was down-regulated in various human tumor cells and tissues. However, miRNA-218 expression was up-regulated in cervical cancer tissues. The results demonstrate that miRNA-944 and miRNA-218 significantly increase in cervical cancer tissues. On the contrary, miRNA-143, miRNA-34A, and miRNA101 in cervical cancer tissues are downregulated. 


\section{CONCLUSION}

The findings of the present work indicate that the expressions of miRNA-143, miRNA-34A, miRNA944, miRNA-101 and miRNA-218 in cervical cancer tissues are different from those of normal tissues. Thus, these miRNAs are suitable as novel biomarkers for early diagnosis of cervical cancer.

\section{DECLARATIONS}

\section{Acknowledgement}

The authors are very grateful to the Second Affiliated Hospital of Zhengzhou University for support for this work

\section{Conflict of Interest}

No conflict of interest associated with this work.

\section{Contribution of Authors}

The authors declare that this work was done by the authors named in this article and all liabilities pertaining to claims relating to the content of this article will be borne by them.

\section{REFERENCES}

1. Jemal A, Bray F, Center MM, Ferlay J, Ward E, Forman D. Global cancer statistics. CA Cancer J Clin 2011; 61: 69-90.

2. Pfaendler KS, Wenzel L, Mechanic MB, Penner KR. Cervical cancer survivorship: long-term quality of life and social support. Clin Ther 2015; 37: 39-48.

3. Vargo JA, Boisen MM, Comerci JT, Kim H, Houser CJ, Sukumvanich $P$. Neoadjuvant radiotherapy with or without chemotherapy followed by extrafascial hysterectomy for locally advanced endometrial cancer clinically extending to the cervix or parametria. Gynecol Oncol 2014; 135: 190-195.

4. Zhang $P$, Yang $B$, Yao $Y Y$, Zhong $L X$, Chen $X Y$, Kong QY, Wu ML, Li C, Li H, LiU J. PIAS3, SHP2 and SOCS3 Expression patterns in Cervical Cancers: Relevance with activation and resveratrol-caused inactivation of STAT3 signaling. Gynecol Oncol 2015; http://dx.doi.org/10.1016/j.ygyno.2015.09.087

5. Moreno-Moya JM, Vilella F, Simon C. MicroRNA: key gene expression regulators, Fertil Steril 2014; 101: $1516 e 1523$.

6. Bartel DP. MicroRNAs: genomics, biogenesis, mechanism, and function, Cell 2004; 116: 281-297.

7. Lopez-Serra P, Esteller M. DNA methylation-associated silencing of tumor suppressor microRNAs in cancer. Oncogene 2012; 31: 1609-1622.
8. Wang N, Zhou $Y$, Zheng L, Li H. MiR-31 is an independent prognostic factor and functions as an oncomir in cervical cancer via targeting ARID1A. Gynecol Oncol 2014; 134: 129-137.

9. World Medical Organization. Declaration of Helsinki. British Medical Journal (7 December) 1996; 313(7070): 1448-1449.

10. Deftereos G, Corrie SR, Feng Q, Morihara J, Stern J, Hawes SE, Kiviat NB. Expression of mir-21 and mir-143 in cervical specimens ranging from histologically normal through to invasive cervical cancer. PLoS One 2011; 6: e28423.

11. Yan $X$, Chen $X$, Liang $H$, Deng $T$, Chen $W$, Zhang S, Liu M, Gao X, Liu Y, Zhao C. miR-143 and miR-145 synergistically regulate ERBB3 to suppress cell proliferation and invasion in breast cancer. Mol Cancer 2014; 13: 220.

12. Liu H, Lin $H$, Zhang L, Sun $Q$, Yuan $G$, Zhang L, Chen S, Chen Z. miR-145 and miR-143 regulate odontoblast differentiation through targeting KIf4 and Osx genes in a feedback loop. J Biol Chem 2013; 288: 9261-9271.

13. Pham $H$, Rodriguez CE, Donald GW, Hertzer KM, Jung $X S$, Chang $H H$, Moro A, Reber HA, Hines OJ, Eibl G. miR-143 decreases COX-2 mRNA stability and expression in pancreatic cancer cells, Biochem Biophys Res Commun 2013; 439: 6-11.

14. Hu CE, Liu YC, Zhang HD, Huang GJ. The RNA-binding protein $P C B P 2$ facilitates gastric carcinoma growth by targeting miR-34a. Biochem Bioph Res Co 2014; 448: 437-442.

15. Yamakuchi M, Lowenstein CJ. MiR-34, SIRT1, and p53: the feedback loop. Cell Cycle 2009; 8: 712-715.

16. Cao W, Fan R, Wang L, Cheng S, Li H, Jiang J, Geng M, Jin $Y, W u Y$. Expression and regulatory function of miRNA-34a in targeting survivin in gastric cancer cells. Tumor Biol 2013; 34: 963-971.

17. Powrózek T, Krawczyk P, Kowalski DM, Winiarczyk K, Olszyna-Serementa M, Milanowski J. Plasma circulating microRNA-944 and microRNA-3662 as potential histologic type-specific early lung cancer biomarkers. Trans/ Res 2015; 166: 315-323.

18. Xie H, Lee L, Scicluna P, Kavak E, Larsson C, Sandberg $R$, Lui WO. Novel functions and targets of miR-944 in human cervical cancer cells. Int J Cancer 2015; 136: E230-241.

19. Lui WO, Pourmand N, Patterson BK, Fire A. Patterns of known and novel small RNAs in human cervical cancer. Cancer Res 2007; 67: 6031-6043.

20. Semaan A, Qazi AM, Seward S, Chamala S, Bryant CS, Kumar $S$, Morris $R$, Steffes $C P$, Bouwman $D L$, Munkarah AR, Weaver DW, Gruber SA, Batchu RB. MicroRNA-101 inhibits growth of epithelial ovarian cancer by relieving chromatin-mediated transcriptional repression of p21 (waf1/cip1). Pharm Res 2011; 28: 3079-3090.

21. Chen MB, Yang L, Lu PH, Fu XL, Zhang Y, Zhu YQ, Tian $Y$. MicroRNA-101 down-regulates sphingosine kinase 1 
in colorectal cancer cells. Biochem Bioph Res Co 2015, 463: 954-960.

22. Zhang J, Liu J, Liu Y, Wu W, Li X, Wu Y, Chen H, Zhang K, Gu L. miR-101 represses lung cancer by inhibiting interaction of fibroblasts and cancer cells by downregulating CXCL12. Biomed Pharmacother 2015; 74: 215-221.

23. Varambally $S$, Cao $Q$, Mani RS, Shankar S, Wang $X$, Ateeq B, Laxman B, Cao X, Jing X, Ramnarayanan $K$. Genomic loss of microRNA-101 leads to overexpression of histone methyltransferase EZH2 in cancer. Science 2008; 322: 1695-1699.

24. Tatarano S, Chiyomaru $T$, Kawakami K, Enokida $H$, Yoshino $H$, Hidaka $H$, Yamasaki $T$, Kawahara $K$,
Nishiyama K, Seki N. miR-218 on the genomic loss region of chromosome $4 p 15.31$ functions as a tumor suppressor in bladder cancer. Int J Oncol 2011; 39: 1321.

25. Zheng $X$, Yang D, Liu X, Wang N, Li B, Cao H, Lu Y, Wei $G$, Zhou $H$, Zheng J. Identification of a new anti-LPS agent, geniposide, from Gardenia jasminoides Ellis, and its ability of direct binding and neutralization of lipopolysaccharide in vitro and in vivo. Int Immunopharmacol 2010; 10: 1209-1219.

26. Yang $L, L i Q$, Wang $Q$, Jiang Z, Zhang L. Silencing of miRNA-218 promotes migration and invasion of breast cancer via Slit2-Robo1 pathway. Biomed Pharmacother 2012; 66: 535-540. 